\title{
Corf-9308229--1
}

\section{NEUTRON MULTIPLICITIES IN SPONTANEOUS FISSION AND NUCLEAR STRUCTURE STUDIES}

J.H. Hamilton, J. Kormicki, Q. Lu, D. Shi, K. Butler-Moore, A.V. Ramayya, W.-C. Ma, B.R.S. Babu

Department of Physics and Astronomy

Vanderbilt University, Nashville, TN, 37235

G.M. Ter-Akopian, Yu. Ts. Oganessian, G.S. Popeko, A.V. Daniel

Joint Institute for Nuclear Research, Dubna, Russia

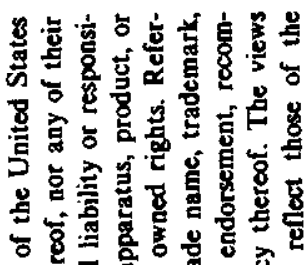

Isinghua University, Beifing, P.K. China

J. Kliman, V. Polhorsky, M. Morhac

Institute of Physics SASc,

Bratislava, Slovak Republic

J.D. Cole, R. Aryaeinejad, R.C. Greenwood

Idaho National Engineering Lab.,

Idaho Falls, ID, 83415

N.R. Johnson, I.Y. Lee, F.K. McGowan

Oak Ridge National Laboratory,

Oak Ridge, TN 37831
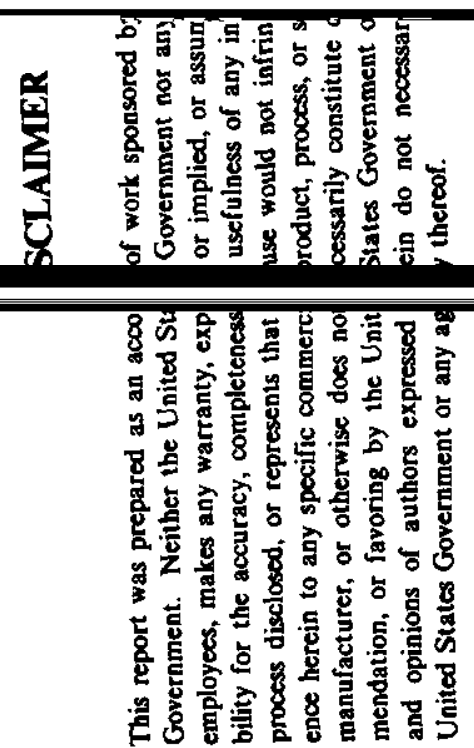

\section{INTRODUCTION}

New insights into the fission process can be gained by better quantitative knowledge of how the energy released in fission is distributed between the kinetic energy of the two fragments, the excitation energy of the two fragments and the number of neutrons emitted. Studies of prompt gamma-rays emitted in spontaneous fission (SF) with large arrays of Compton suppressed Ge detector arrays are providing new quantitative answers to longstanding questions concerning fission as well as new insights into the structure of neutronrich nuclei. For the first time the triple gamma coincidence technique was employed in spontaneous fission studies. Studies of $\mathrm{SF}$ of ${ }^{252} \mathrm{Cf}$ and ${ }^{242} \mathrm{Pu}$ have been carried out. 
These $\gamma-\gamma-\gamma$ data provide powerful ways to identify uniquely gamma rays from a particular nucleus in the very complex gamma-ray spectra given off by the over 100 different nuclei produced. The emphasis of this paper is on the first quantitative measurements of the multiplicities of the neutrons emitted in SF and the energy levels populated in the fragments. Indeed, in the break up into Mo-Ba pairs, we have identified for the first time fragments associated with from zero up to ten neutrons emitted and observed the excited energy states populated in these nuclei. The zero neutron emission pairs like ${ }^{104} \mathrm{Mo}$ ${ }^{148} \mathrm{Ba},{ }^{106} \mathrm{Mo} \cdot{ }^{146} \mathrm{Ba}$ and ${ }^{104} \mathrm{Zr}-{ }^{148} \mathrm{Ce}$ observed in this work are particularly interesting because they represent a type of cold fission or a new mode of cluster radioactivity as proposed by Greiner, Sandulescu and co-workers ${ }^{1,2}$. These data provide new insights into the processes of cluster radioactivity and cold fission.

These SF studies also continue to be a rich source of information on neutron rich nuclei. The triple gamma coincidence technique was used to uniquely identify levels in ${ }^{136} \mathrm{Te}$ and new higher spin states in its $\mathrm{N}=84$ isotones ${ }^{138} \mathrm{Xe}$ and ${ }^{140} \mathrm{Ba} .{ }^{3}$ Earlier evidence for octupole deformation was observed in the even-even $144,146 \mathrm{Ba}^{4}$. Recently we have discovered similar evidence for octupole deformation odd- $\mathrm{A}$ in ${ }^{143} \mathrm{Ba}$ and observed a new band to high spin in ${ }^{145} \mathrm{Ba}^{5}$. These nuclei can give more direct information on the orbitals involved in the octupole deformation.

\section{EXPERIMENTAL METHODS}

A spontaneous fission source of ${ }^{252} \mathrm{Cf}(0.1 \mathrm{\mu g})$ with a strength of about $6 \times 10^{4}$ fissions/sec and with a $250 \mu \mathrm{m} \mathrm{Be}$ window was used for the Oak Ridge study. This source was placed in the center of the 20 Compton-suppressed Ge-detector Compact Ball at the Holifield Heavy Ion Research Facility at Oak Ridge National Laboratory. Approximately $2 \times 10^{9} \gamma-\gamma$ coincidences were collected during a five day run. Both double- and triple-coincidence events were recorded and sorted in data analysis. A comparable strength source and the same experimental arrangements were used to study the SF of ${ }^{242} \mathrm{Pu}$. The coincidences obtained for the $\gamma$-rays of fission fragment pairs can be used as triggers for the fission events of a given nucleus against the background of $\beta$ decay and fission of other nuclides. The triple coincidence technique is particularly powerful in eliminating or reducing all types of background, especially those produced by the process of two or more gamma-rays from different nuclei falling in an energy gate set on a single $\gamma$-ray in a $\gamma-\gamma$ coincidence experiment.

\section{NEUTRON MULTIPLICITIES AND COLD FISSIONS (CLUSTER RADIOACTIVITY)}

The distribution (relative intensities) of the different-mass partners formed through the emission of different numbers of neutrons are of particular interest in understanding the fission process. Of special interest is the relative probability of zero neutron emission. This case may correspond to a new type of cluster radioactivity. The phenomena of cluster radioactivity was initiated by (Sandulescu, Poenaru and Greiner ${ }^{6}$ who proposed this new class of radioactivity in which a nucleus decays into two fragments with both masses heavier than the alpha particle but one lighter than the usual fission fragments without the emission of other iight particles like neutrons. Greiner, Ivascu, Poenaru and Sandulescu ${ }^{1}$ have recently reviewed this field. Greiner and Sandulescu ${ }^{2}$ have emphasized that another prediction of their two-center shell is cold fission in addition to cluster radioactivity. In this case a nucleus splits into two "unexcited" nuclei. Cold fission means both fragments are left in low excited states (small excitation energy) so neutron emission is not allowed. The energy goes mainly into kinetic energy of the fragments. 
In induced fission Gönnerwein and his colleagues (see elsewhere in these proceeding) have observed events in which the kinetic energy of the fragments essentially equal the $Q$ value. In our $S F$ fission studies we carry this evidence one step further.

The ${ }^{252} \mathrm{Cf}$ SF data have been analyzed to determine the different neutron emission channels and the relative intensities of these channels. The procedure is to gate on a gamma transition in for example ${ }^{148} \mathrm{Ce}$ and look for the gamma rays associated with the different mass zirconium isotopes. The difference between the masses of the two partners and $A=252$ gives the number of neutrons emitted. In Fig. 1 is shown the low energy region of the gamma ray spectrum in coincidence with the first six transitions in the yrast cascade in ${ }^{148} \mathrm{Ce}$. Gamma rays corresponding to transitions in ${ }^{104} \mathrm{Zr}$ down to ${ }^{98} \mathrm{Zr}$ are observed in coincidence with ${ }^{148} \mathrm{Ce}$. Then gates were set on each zirconium isotope and the transitions in the different cerium isotopes were observed.

To establish that a particular partner pair was being observed, the right transition had to be seen in the other partner when pulling gates from each partner. Table 1 and Fig. 2. show the mass distributions of $\mathrm{Zr}$ isotopes obtained in coincidence with ${ }^{146} \mathrm{Ce}$ and ${ }^{148} \mathrm{Ce}$. These distributions are deduced from the detected intensities of transitions between the lowest excited and ground states of the $\mathrm{Zr}$ fragments corrected for the known $\gamma$-ray energy dependence of the detection efficiency $\varepsilon\left(E_{\gamma}\right)$ of the Close-packed Ge detector Ball. Including the small variation of the $\gamma$-ray multiplicity with the mass number of the $\mathrm{Zr}$ fragments, we deduced from the data of Table 1 that the most probablis $\mathrm{Zr}$ mass numbers are shifted by two mass units (from ${ }^{100} \mathrm{Zr}$ to ${ }^{102} \mathrm{Zr}$ ) in going from ${ }^{148} \mathrm{Ce}$ to ${ }^{146} \mathrm{Ce}$, whereas the mean value of the $\mathrm{Zr}$ fission fragment mass varies rather slowly, 0.5 mass units between ${ }^{148} \mathrm{Ce}$ and ${ }^{146} \mathrm{Ce}$. This comes about because of the difference in the intensities of the higher and lower neutron emission channels in the two nuclei as seen in Fig. 2.

Using the same gating procedures as outlined above, a complete set of relative yields of different pairs of fission fragments is presented in Table 2 for the charge division of ${ }^{252} \mathrm{Cf}$ into $\mathrm{Z}_{\mathrm{L}} / \mathrm{Z}_{\mathrm{H}}=42 / 56(\mathrm{Mo} / \mathrm{Ba})$. This is the first time such detailed distributions as those given in Tables 1 and 2 have been obtained in a fission study. The last row and column of Table 2 give the sums of the correlated relative independent yields of Mo and $\mathrm{Ba}$ isotopes which are the independent yields according to the known evaluated data tables. ${ }^{7}$ The distributions presented in Tables 1 and 2 give us a deeper insight into the multiplicity distributions of prompt fission neutrons. Note the ${ }^{106} \mathrm{Mo}-{ }^{146} \mathrm{Ba}$ and ${ }^{104} \mathrm{Mo}$ ${ }^{148} \mathrm{Ba}$ pairs have zero neutron emission, and the ${ }^{104} \mathrm{Mo}-{ }^{138} \mathrm{Ba}$ pair has 10 neutron emission. Figure 2 shows the neutron multiplicity distributions obtained in coincidence with ${ }^{148} \mathrm{Ce}$ and ${ }^{146} \mathrm{Ce}$ fission fragments, and Fig. 3 shows the neutron multiplicity distribution for the $\mathrm{Mo} / \mathrm{Ba}$ charge division of the californium nucleus. These are the real distributions deduced directly from our experimental data corrected only for the $\gamma$-ray detection efficiency. This is a specific positive feature of our results. Neutron multiplicities obtained previously were deduced from the experimental data via a sophisticated reduction procedure. For the first time we obtained clear data about the long sought fractions of californium spontaneous fission events with zero on to ten prompt neutrons. For the first time the charge asymmetry dependence of the mean number of prompt neutrons can be deduced directly from an experiment. For the 42/56 charge asymmetry we obtained the average neutron multiplicity $\langle U\rangle=3.85$. Similar data for the various fragments pairs are currently being extracted.

Three definite zero neutron emission channels have been observed, ${ }^{104} \mathrm{Zr}-{ }^{148} \mathrm{Ce}$, ${ }^{104} \mathrm{Mo}-{ }^{148} \mathrm{Ba}$ and ${ }^{106} \mathrm{Mo}-{ }^{146} \mathrm{Ba}$. The energy levels of these nuclei observed in this work are shown in Fig. 4 and 5. The single neutron binding energies are given below each nucleus. From momentum conservation, the kinetic energies are roughly equivalent for each fragment. Since in these channels zero neutrons are emitted, the nuclei must be left after fission with less than the neutron binding energies which vary from 4.7 to 7.7 


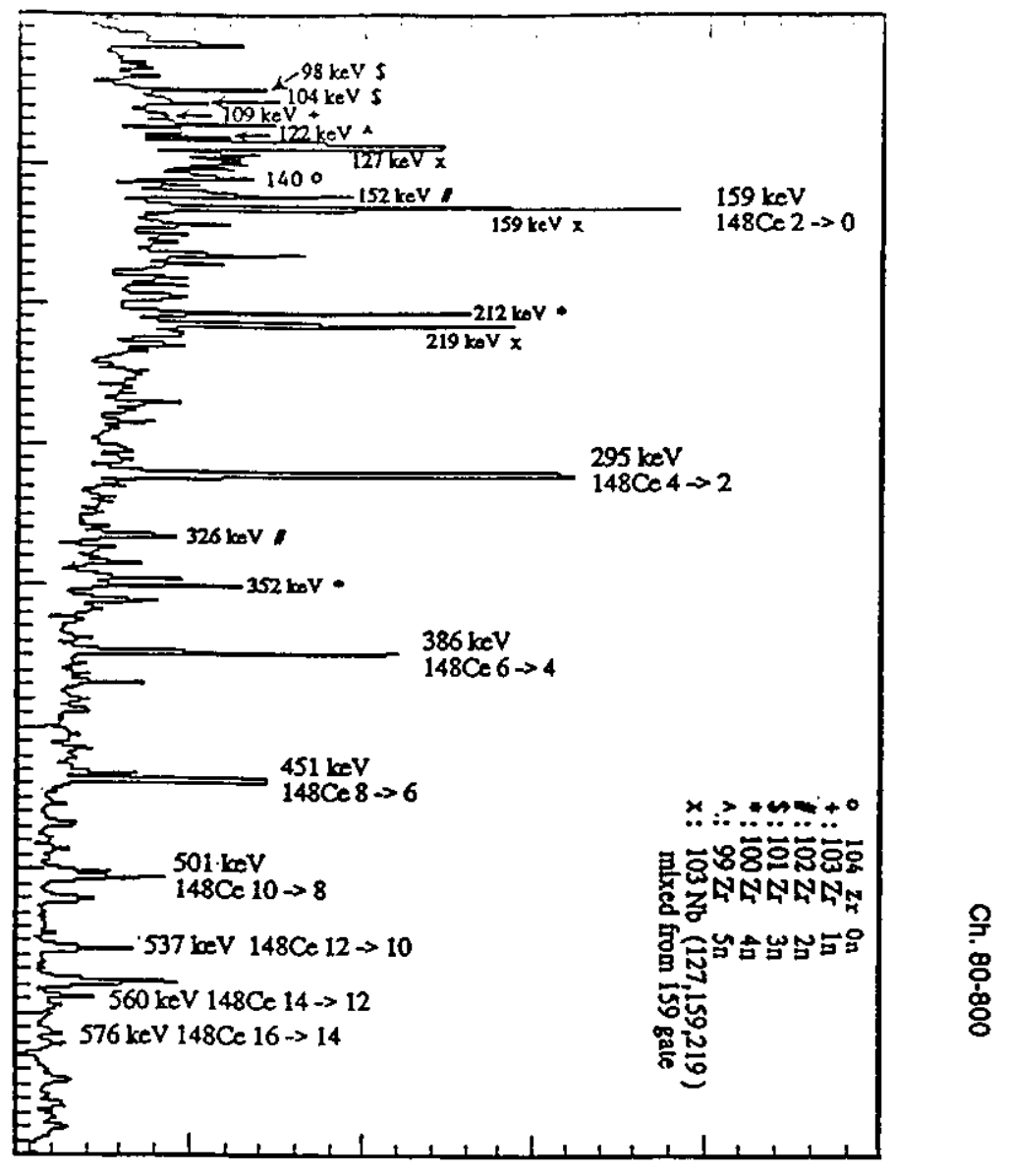

Figure 1. Gamma rays in coincidence with sum of yrast cascades.

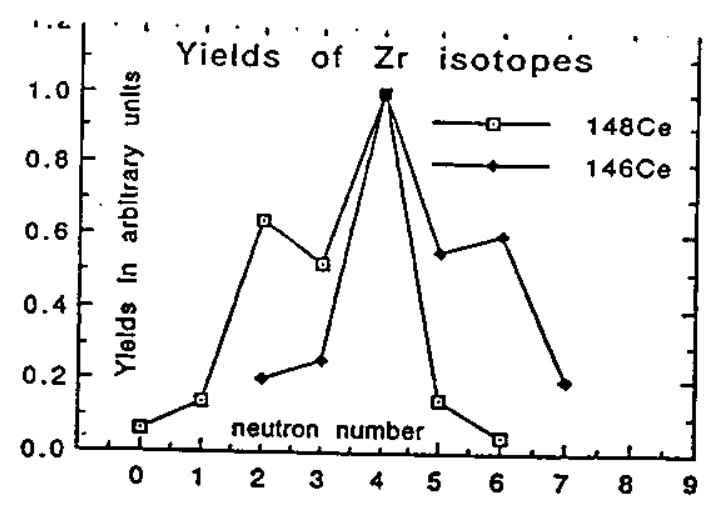

Figure 2. = Table 1 .

Table 1. Normalized relative yields of the correlated fragment pairs for $Z_{L} / Z_{H}=40 / 58$.

\begin{tabular}{ccccccccc}
\hline \hline $\begin{array}{c}\mathrm{Ce} \\
\text { partner }\end{array}$ & $\mathrm{Zr}^{98}$ & $\mathrm{Zr}^{99}$ & $\mathrm{Zr}^{100}$ & $\mathrm{Zr}^{101}$ & $\mathrm{Zr}^{102}$ & $\mathrm{Zr}^{103}$ & $\mathrm{Zr}^{104}$ & $<\mathrm{A}>$ \\
\hline${ }^{148} \mathrm{Ce}$ & 0.05 & 0.15 & 1.00 & 0.52 & 0.64 & 0.14 & 0.06 & 100.9 \\
${ }^{146} \mathrm{Ce}$ & & 0.20 & 0.60 & 0.55 & 1.00 & 0.26 & 0.20 & 101.4 \\
\hline \hline
\end{tabular}


Table 2. Reiative yields of the correlated fragment pair masses $Y\left(A_{L}, A_{H}\right)$ for $\mathrm{Z}_{\mathrm{L}} / \mathrm{Z}_{\mathrm{H}}=42 / 56$. The matrix is normalized to 100 .

\begin{tabular}{cccccccc}
\hline & ${ }^{138} \mathrm{Ba}$ & $140 \mathrm{Ba}$ & ${ }^{142} \mathrm{Ba}$ & ${ }^{144} \mathrm{Ba}$ & ${ }^{146} \mathrm{Ba}$ & ${ }^{148} \mathrm{Ba}$ & $\Sigma$ \\
\hline${ }^{100} \mathrm{Mo}$ & & & $<0.4$ & $<0.4$ & $<0.4$ & $<0.6$ & \\
${ }^{102} \mathrm{Mo}$ & & $<0.4$ & 0.6 & 2.9 & 4.7 & 1.7 & 9.9 \\
${ }^{104} \mathrm{Mo}$ & $0.2(1)$ & 0.9 & 7.9 & 26.2 & 8.2 & 1.8 & 45.2 \\
${ }^{106} \mathrm{Mo}$ & 0.5 & 5.3 & 16.8 & 12.3 & 0.1 & & 35.1 \\
${ }^{108} \mathrm{Mo}$ & 0.2 & 4.7 & 3.0 & 1.8 & & & 9.6 \\
$\Sigma$ & 0.9 & 10.9 & 28.3 & 41.5 & 13.0 & 3.5 & \\
\hline \hline
\end{tabular}

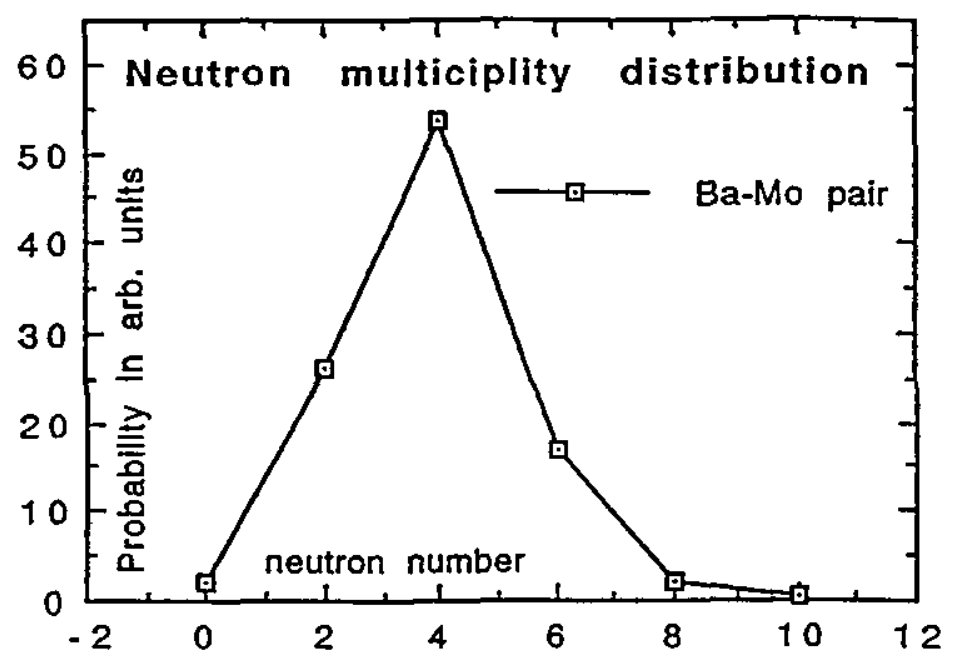

Figure 3. Relative neutron distribution for Ba-Mo pairs.

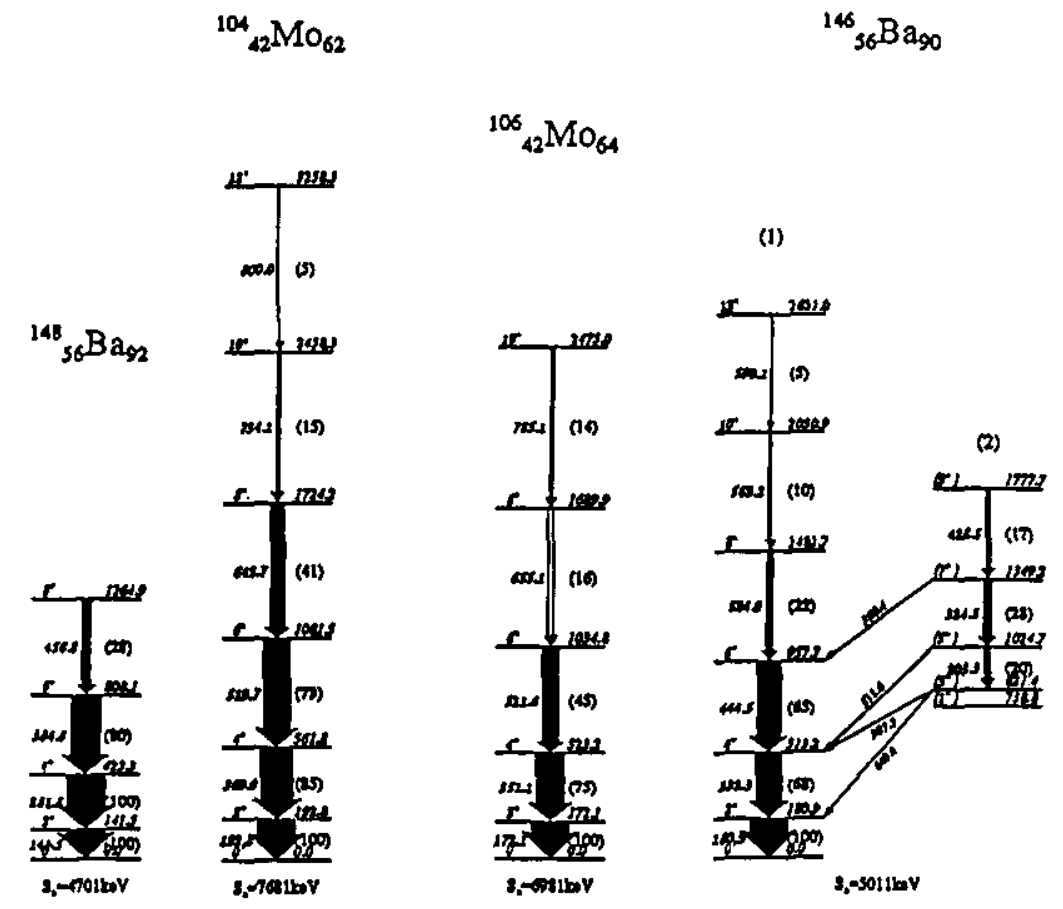

Figure 4. Levels observed in zero neutron emission (cold fission) correlated pairs ${ }^{108} \mathrm{Mo}-{ }^{148} \mathrm{Ba}$ and ${ }^{106} \mathrm{Mo} \cdot{ }^{146} \mathrm{Ba}$. 
$\mathrm{MeV}$. The large fission energy observed in the $4 \mathrm{n}$ up to 10n channels thus must essentially all go into kinetic energy of the fragments. Note in Figs. 4, 5, the maximum excited state energies observed in these nuclei go from 1265 up to $3558 \mathrm{keV}$ so a small fraction of the fission energy goes into excitation of the low lying excited states in these nuclei with zero neutron emission. Thus these three cases correspond to cold fission or a new type of cluster radioactivity as earlier predicted by the Frankfurt-Bucharest group. 1,2 Earlier in cluster radioactivity, decays to excited states of the heavy fragment where observed. Here, however, we see cluster radioactivity or cold fission where both fragments are left in relatively low energy excited states.

\section{MEAN ANGULAR MOMENTUM DISTRIBUTION}

The intensities of the transitions within the yrast cascade, normalized to 100 for the $2^{+} \rightarrow 0^{+}$transitions, are shown in Table 3 for some complementary fission fragments in the $\mathrm{SF}$ of ${ }^{252} \mathrm{Cf}$, namely $\mathrm{Zr}$ - $\mathrm{Ce}(\mathrm{Zr} 40,58)$ and $\mathrm{Mo}-\mathrm{Ba}(\mathrm{Zr} \mathrm{42,56)}$. From these data on the population of the states as a function of angular momentum, one can extract the average values of the angular momentum $\langle j\rangle$ for the levels populated in each nucleus. For the first two rows ${ }^{146.148} \mathrm{Ce}$, the data represent average over all neutron channels to different $\mathrm{Zr}$ isotopes for the population intensities in these two nuclei. The next two rows give data for ${ }^{148} \mathrm{Ce}$ for specific four and two neutron-out channels to ${ }^{100} \mathrm{Zr}$ and ${ }^{102} \mathrm{Zr}$, respectively. These data are obtained from the $\gamma-\gamma-\gamma$ data by double gating on the $2-0$ transition in ${ }^{100} \mathrm{Zr}$ or ${ }^{102} \mathrm{Zr}$ and the $2-0$ transition in ${ }^{148} \mathrm{Ce}$. The next six rows were then from spectra double gated on the 2-0 transition in each partner. Table 3 gives the angular moments of different specific fission fragments in coincidence with the individual partner nuclei associated with different numbers of emitted neutrons. Our data give a better detailed picture of the correlation of the angular momentum with the mass and $\mathrm{Z}$ of the fission fragments compared to other results. ${ }^{8-11}$ These data are directly related to the initial fragment excitation or, in other words, to the elongation of the fissioning nucleus and neck of the two fragments at the scission point.

\section{LEVELS IN ${ }^{136}$ Te AND HIGH SPIN STATES IN NEUTRON-RICH N=84 ISOTONES}

Nuclei near the doubly magic ${ }_{52}^{132} \mathrm{Sn}_{82}$ are especially interesting with regard to the coupling of the single particle states to the collective deformation. The light $\mathrm{N}=84$ isotones with two neutrons outside the $\mathrm{N}=82$ closed shell are produced in ${ }^{252} \mathrm{CF} S \mathrm{SF}$. Recently we reported the levels in ${ }^{136} \mathrm{Te}$ together with a new high-spin states in the $\mathrm{N}=84$ isotones, ${ }^{138} \mathrm{Xe}$ and ${ }^{140} \mathrm{Ba} .{ }^{[3]}$ The level in ${ }^{136} \mathrm{Te}$ were simultaneously reported by Cizewski et al. ${ }^{12}$ At the same time we studied levels in the ${ }^{112-116} \mathrm{Pd}$ partners. ${ }^{[13]}$

When ${ }^{252} \mathrm{Cf}$ scissions into a heavy fragment and a light fragment, approximately four neutrons and approximately ten $\gamma$ rays are emitted on the average per scission. All the 


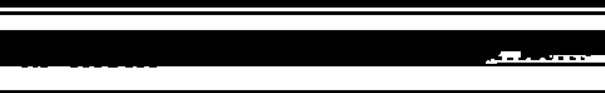

Vic $v$ enuts are in parentureses.

\begin{tabular}{llllllll}
\hline $\begin{array}{l}\text { Fission } \\
\text { fragment }\end{array}$ & $4^{+}-2^{+}$ & $6^{+} 4^{+}$ & $8^{+}-6^{+}$ & $10^{+}-8^{+}$ & $12^{+}-10^{+}$ & $14^{+} \cdot 12^{+}$ & $<j>$ \\
\hline${ }^{146} \mathrm{Ce}^{1)}$ & $100(5)$ & $41(3)$ & $11(2)$ & $4(2)$ & & & $5.1(1.2)$ \\
${ }^{148} \mathrm{Ce}^{1)}$ & $100(5)$ & $85(5)$ & $58(4)$ & $36(4)$ & $21(2)$ & $7(3)$ & $8.1(1.3)$ \\
${ }^{148} \mathrm{Ce}^{2)}$ & $100(5)$ & $78(4)$ & $46(3)$ & $36(3)$ & $20(2)$ & $9(4)$ & $7.8(1.2)$ \\
${ }^{148} \mathrm{Ce}^{3)}$ & $100(5)$ & $75(4)$ & $46(3)$ & $31(3)$ & $19(2)$ & $7(3)$ & $7.7(1.2)$ \\
${ }^{100} \mathrm{Zr}^{4)}$ & $72(4)$ & $61(3)$ & $21(3)$ & & & & $5.1(0.9)$ \\
$\left.{ }^{100} \mathrm{Zr}^{5}\right)$ & $77(4)$ & $70(4)$ & $26(3)$ & & & & $5.5(0.9)$ \\
${ }^{102} \mathrm{Zr}^{4)}$ & $78(4)$ & $50(4)$ & $21(3)$ & & & $5.0(0.9)$ \\
${ }^{102} \mathrm{Zr}^{5)}$ & $92(5)$ & $61(4)$ & $24(3)$ & $5(2)$ & & $5.6(1.0)$ \\
${ }^{144} \mathrm{Ba}^{6)}$ & $92(6)$ & $85(7)$ & $52(6)$ & $15(5)$ & & $6.7(1.4)$ \\
$-104 \mathrm{Mo}^{7)}$ & $76(6)$ & $48(5)$ & $15(5)$ & $7(3)$ & & & $4.9(1.5)$ \\
\hline \hline
\end{tabular}

1) Average over $\mathrm{Zr}$ isotopes (in this case, the gate is the $2^{+}-0^{+}$transition in ${ }^{146,148} \mathrm{Ce}$, respectively), ${ }^{2)}$ with ${ }^{100} \mathrm{Zr}$, ${ }^{3)}$ with ${ }^{102} \mathrm{Zr},{ }^{4)}$ with ${ }^{148} \mathrm{Ce}$, ${ }^{6}$ with ${ }^{104} \mathrm{Mo}$, ${ }^{7)}$ with ${ }^{144} \mathrm{Ba}$, 2-7) these results are derived from two-gates in the triple coincidence spectra (see text).

transitions of a particle light fragment and those of its complementary heavy partners (and vice-versa) are in coincidence with each other. So, coincidence spectra from gating a transition in a particular nuclide exhibits peaks not only for the transitions in that nuclide, but also for those in its several heavy or light fission partners. In addition there is a high probability that a gate on a particular energy transition will overlay not only the transition of interest, but nearly the same energy transitions (one or more) in different isotopes. These complexities make it difficult to identify unknown transitions in new nuclides in doublemenincidence conecteon

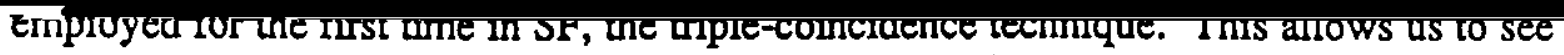
only the transitions in the two fission partners or emphasize the transitions in one partner. The triple $\gamma$-ray coincidence technique has been employed in SF studies to identify uniquely states in ${ }^{136} \mathrm{Te}$, and also to resolve doublets in ${ }^{138} \mathrm{Xe}$ and ${ }^{140} \mathrm{Ba}$. Let us illustrate the power of this technique.

Figure 6 shows three double coincidence spectra. The top spectrum is gated on the $2^{+} \rightarrow 0^{+}$transition in ${ }^{114} \mathrm{Pd}$, and $2 \mathrm{n}$ complement to ${ }^{136} \mathrm{Te}$. The middle spectrum is gated on the $2^{+} \rightarrow 0^{+}$transition in ${ }^{112} \mathrm{Pd}$, the $4 \mathrm{n}$ complement of ${ }^{136} \mathrm{Te}$. In both the $\mathrm{Pd}$ gates we see other Pd yrast transitions and several peaks that are common in both these gates, these are transitions in $\mathrm{Te}$ isotopes. The bottom spectrum is gated on the most intense peak in common with the top and middle spectra, the $607 \mathrm{keV}$ transition. In this gate we see $\gamma$ rays of ${ }^{112,114} \mathrm{Pd}$ and candidate $\gamma$-rays for transitions in ${ }^{136} \mathrm{Te}$. However, in all three spectra there are a number of unidentified peaks. Figure 7 includes three triple coincidence spectra. The top two are gated with the $607 \mathrm{keV}$ transition assigned to ${ }^{136} \mathrm{Te}$ and the $2^{+} \rightarrow 0^{+}$transitions of its ${ }^{112,114} \mathrm{Pd}$ complements. The bottom spectrum consists of gates on two of the transitions newly assigned to ${ }^{136} \mathrm{Te}$. The other transitions in ${ }^{136} \mathrm{Te}$ are now seen as prominent peaks. The same new transitions in ${ }^{136} \mathrm{Te}$ were 


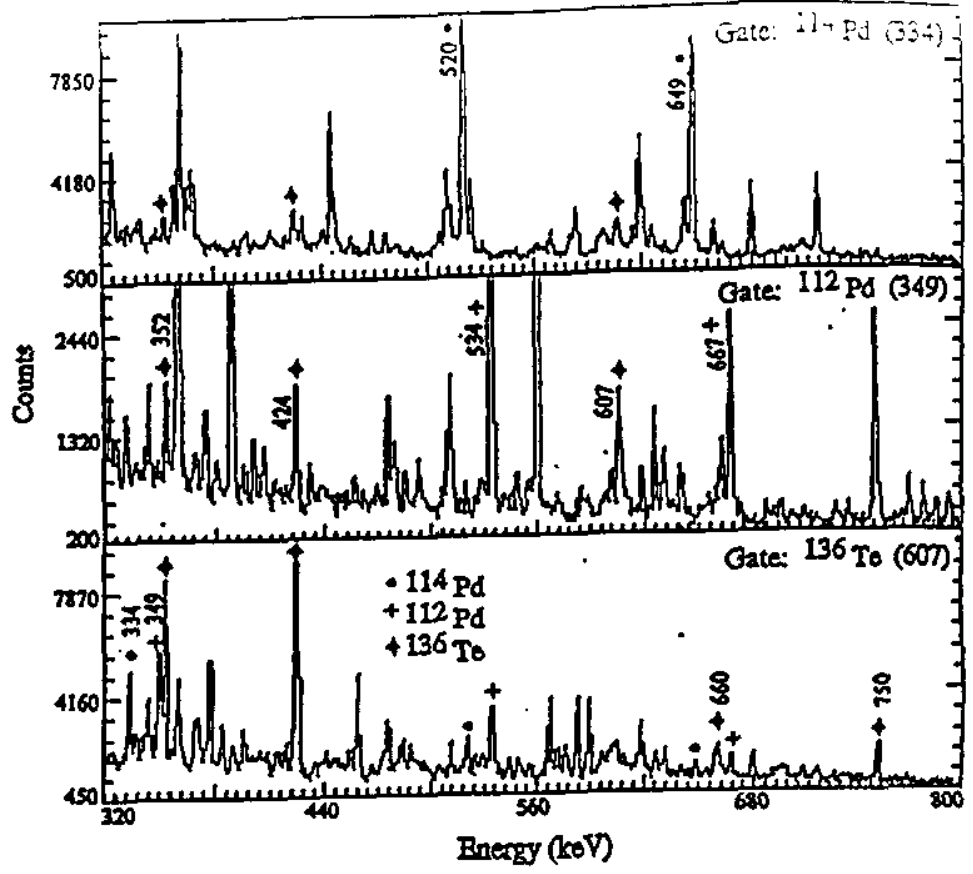

Figure 6. Spectra in coincidence with single gamma ray in one nucleus as shown.

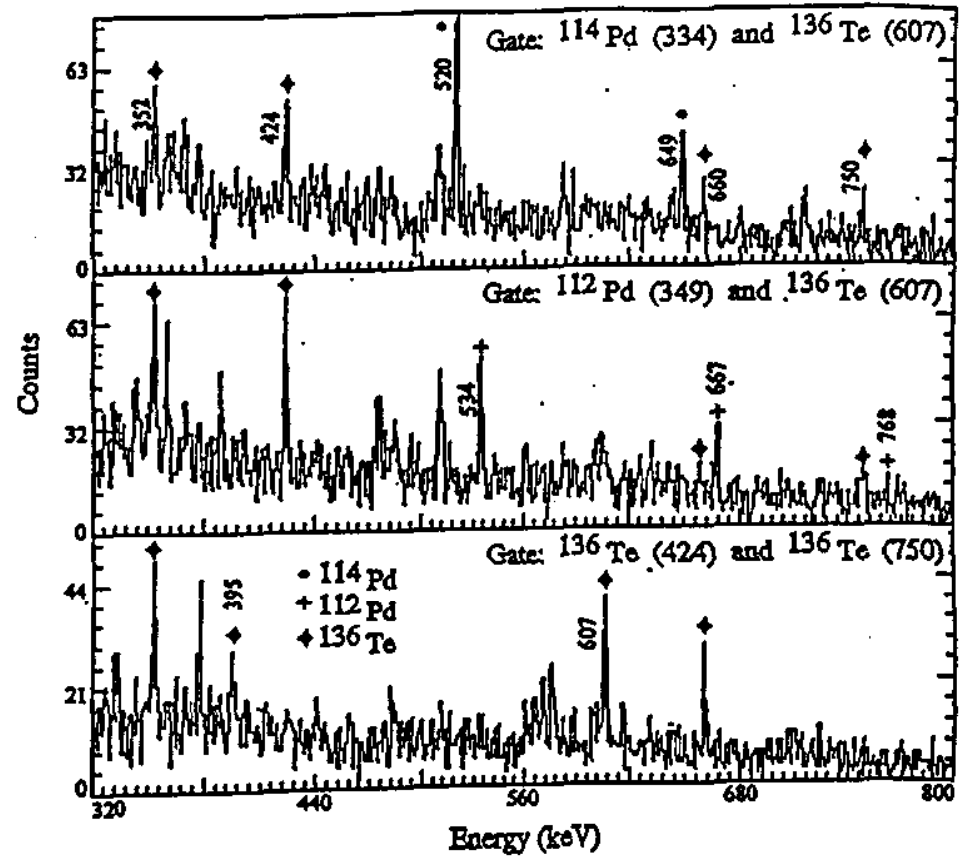

Figure 7. Spectra in coincidence with double gated gamma rays as shown.

simultaneously reported first by Cizewski et al. ${ }^{12}$ However, in that work, the doublet nature of the $423 \mathrm{keV}$ in the double-coincidence spectra prevented them from obtaining a precise energy for the $4^{+}$and higher levels in ${ }^{136} \mathrm{Te}$.

The level schemes of ${ }^{138} \mathrm{Xe}$ and ${ }^{140} \mathrm{Ba}$, known previously only up to $4^{+}$, were extended to $12^{+}$and $8^{+}$, respectively. These are shown in Fig. 8. Note that in both ${ }^{138} \mathrm{Xe}$ and ${ }^{140} \mathrm{Ba}$ the $6^{+} \rightarrow 4^{+}$and $4^{+} \rightarrow 2^{+}$transitions are very close doublets in energy. These doublets are established clearly in the respective isotopes with the triple coincidence data. Another interesting feature in the high spin data of ${ }^{136} \mathrm{Te}$ and ${ }^{138} \mathrm{Xe}$ is the energies of the $8^{+} \rightarrow 6^{+}$transitions. These transitions have energies significantly greater than the energies of either the $10^{+} \rightarrow 8^{+}$or the $6^{+} \rightarrow 4^{+}$transitions in these two nuclei. This indicates a definite change in structure between the $6^{+}$to $8^{+}$levels in these 
nuclei.

$12+354$

$\underline{12+3188}$

$10+2972$

$10+202$

$\underline{8+} 2469$

$8+2084$

$8+213$

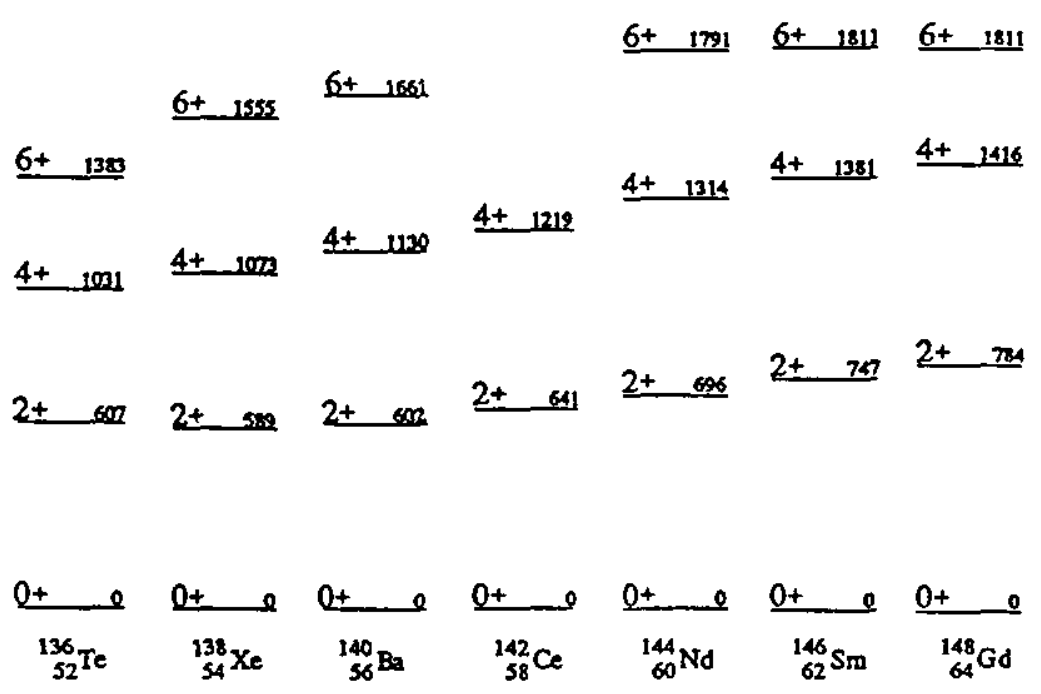

Figure 8. Levels in $N=84$ isotone. The levels in ${ }^{136} \mathrm{Te}$ and above $4^{+}$ in ${ }^{98} \mathrm{Xe}$ and ${ }^{140} \mathrm{Ba}$ are new.

\section{BAND CROSSING IN NEUTRON-RICH Pd ISOTOPES}

Another example of the detailed physics one can obtain is our observation of new excited states and band crossings in ${ }^{112} \mathrm{Pd},{ }^{114} \mathrm{Pd}$ and ${ }^{116} \mathrm{Pd}^{13}$. In the previous experiments the levels up to spin $\mathrm{J}^{\pi}=6^{+}$have been reported in these isotopes. Now we have extended the excited states of ${ }^{112} \mathrm{Pd},{ }^{114} \mathrm{Pd}$ and ${ }^{116} \mathrm{Pd}$ to $10^{+}, 12^{+}$and $12^{+}$, respectively.

Shown in Fig. 6 single gamma gates contain peaks not only in coincidence with yrast transitions, but also in coincidence with several $\gamma$-rays in complementary Te fragments, as well as with transitions in other isotopes with the overlapping gates. To eliminate most of the $\gamma$-rays not associated with the isotopes of interest, we employed the triplecoincidence technique as discussed above. The level schemes of ${ }^{112} \mathrm{Pd}$, ${ }^{14} \mathrm{Pd}$, deduced from our data, are shown in Fig. 9 with identified excited states extended to $10^{+}, 12^{+}$and $12^{+}$, respectively. We carried out cranked shell model (CMS) calculations to determine whether the proton orbital $\left(\pi g_{9 / 2}\right)$ or neutron orbital $\left(\mathrm{vh}_{11 / 2}\right)$ is responsible for band crossings at the observed rotational frequencies. The calculations predict a band crossing related to the alignment of two $g_{92}$ protons at $\hbar \omega=0.35 \mathrm{MeV}$ which is very close to the experimental values of $0.32-0.34$ in ${ }^{112,114} \mathrm{Pd}$. The crossing related to the alignment of two $h_{11 / 2}$ neutrons is predicted to occur at $\hbar \omega=0.42 \mathrm{MeV}$, which is much higher than the observed values. Hence, we propose that the $\mathrm{g}_{9 / 2}$ proton orbital is responsible for backbending in this region. 


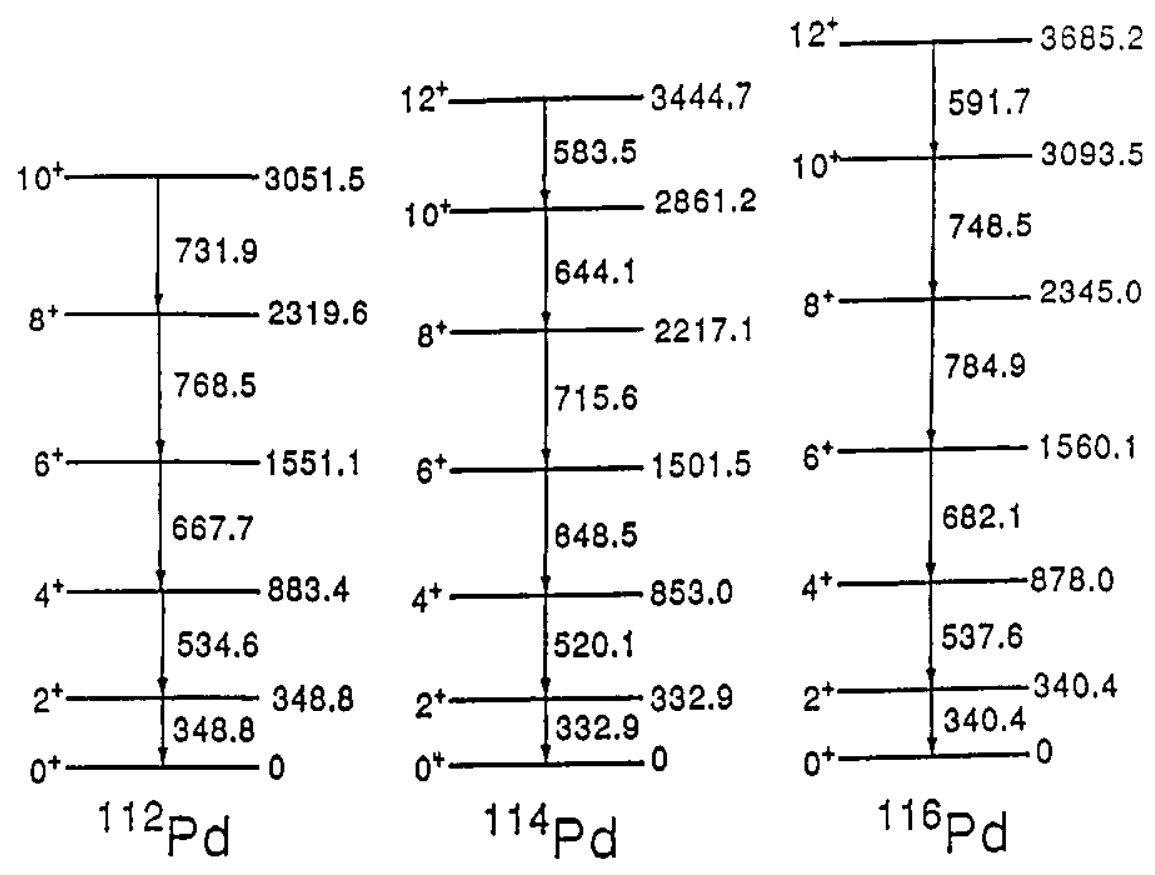

Figure 9. Levels in ${ }^{112-116} \mathrm{~Pb}$. The levels above $6^{+}$are new.

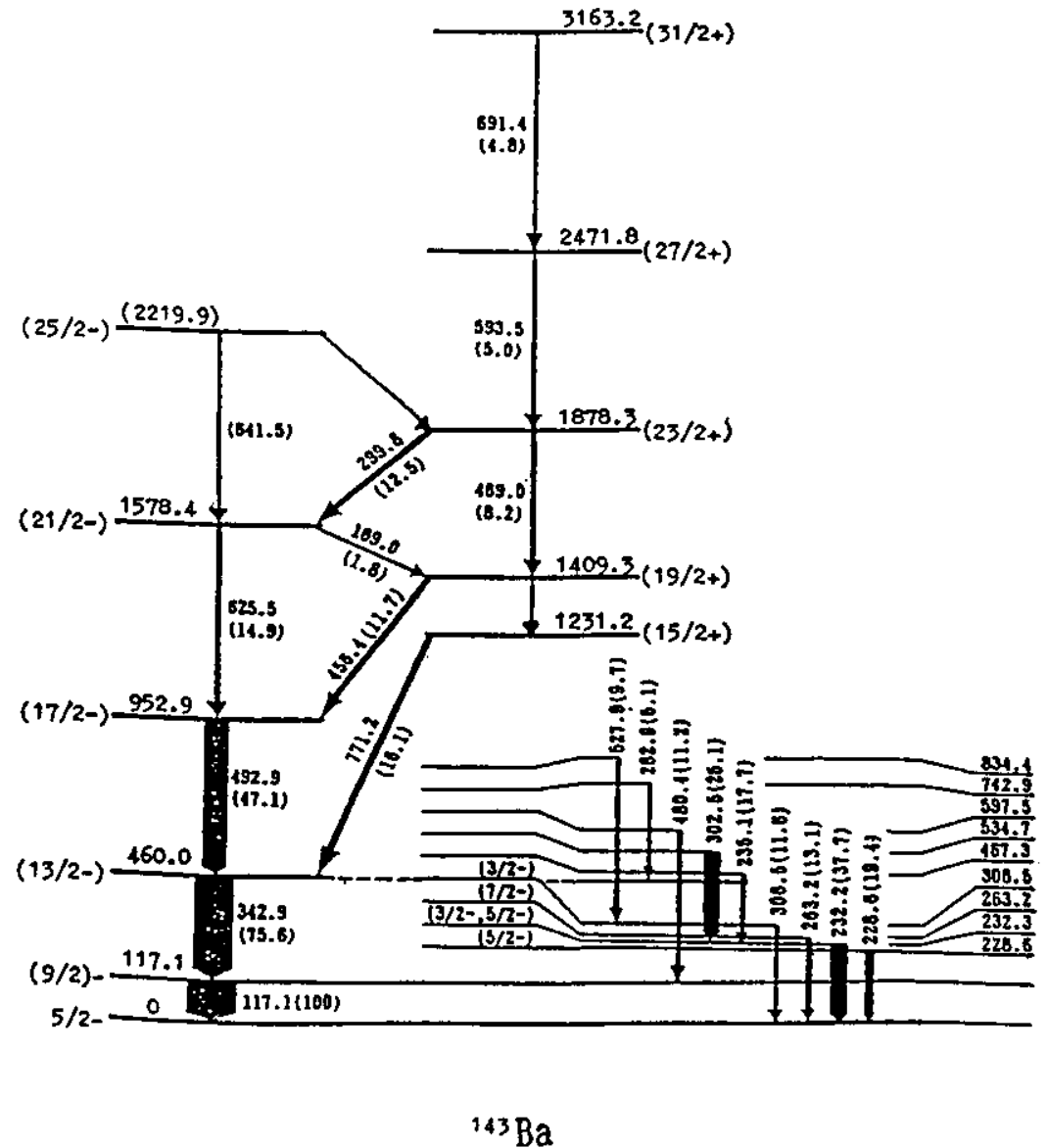

Figure 10. New levels reported above $9 / 2-117.1 \mathrm{keV}$ level that indicate octupole deformations in ${ }^{143} \mathrm{Ba}$.

\section{OCTUPOLE DEFORMATION IN Ba NUCLEI}

From studies of spontaneous fission of ${ }^{252} \mathrm{Cf}$, Phillip et al ${ }^{4}$ found evidence for two intertwining positive and negative parity levels connected by fast $\mathrm{E} 1$ transitions in ${ }^{144,146} \mathrm{Ba}$ as shown in Fig. 4 for ${ }^{146} \mathrm{Ba}$. Their level patterns and $\mathrm{E} 1$ transition rates indicated evidence for the onset of octupole deformation with increasing spin. 
We have studied the level structure of odd $\mathrm{A}^{143} \mathrm{Ba}$ from the spontaneous fission of ${ }^{252} \mathrm{Cf}$. New level structures are found built on the known $92^{\circ}$ level at $117 \mathrm{keV}$ as shown in Fig. 10. One cannot help but be struck by the strong similarity of these new intertwined levels in ${ }^{143} \mathrm{Ba}$ and the intertwined positive and negative parity levels in ${ }^{144} \mathrm{Ba}$. Note ${ }^{142} \mathrm{Ba}$ does not exhibit the simple two intertwined band structure of ${ }^{144} \mathrm{Ba}$. In ${ }^{143} \mathrm{Ba}$ already by the $1409.3 \mathrm{keV}$ level, tentatively assigned $\left(19 / 2^{+}\right)$one sees level spacings characteristics of a band associated with the rotation of an octupole deformed shape. This suggests that the odd neutron is important in helping drive the octupole deformation in ${ }^{43} \mathrm{Ba}$.

\section{ACKNOWLEDGEMENT}

I.Y. Lee is now at Lawrence Berkeley Laboratory. Work is supported at Vanderbilt University by U.S. Department of Energy under grant No. DE-FG-05-88ER40407, at Idaho by U.S. Department of Energy under contract No. DE-AC07-76ID01570 and at Oak Ridge National Laboratory by Martin Marietta Energy Systems under contract No. DEAC05840R21400 with the U.S. Department of Energy.

\section{REFERENCES}

1. W. Greiner, M. Ivascu, D. N. Poenaru, A. Sandulescu, "Treaties on Heavy Ion Science, Vol. 8, Nuclei Far From Stability", D.A. Bromley, ed., Plenum Press (1989).

2. W. Greiner and A. Sandulescu, Scientific American, 262:58 (1990).

3. K. Butler-Moore, J.H. Hamilton, A.V. Ramayya, S. Shu, X. Zhao, W. C. Ma, J. Kormicki, J.K. Deng, W.B. Gao, J. D. Cole, R. Aryaheinejad, I.Y. Lee, N.R. Johnson, F.K. McGowan, G. Ter-Akopian and Y. Oganessian, J. Phys. G: Nucl. Part. Phys. 19:L121-L126 (1993).

4. W. R. Phillips et al. Phys. Rev. Lett. 57:3257 (1986).

5. S.Zhu, D.B. Wang, Q.H. Lu, D. Shi, J.H. Hamilton, W.C. Ma, A.V. Ramayya, R.S. Babu, G.M. Ter-Akopian, Yu. Ts. Oganesian, J.D. Cole, R. Aryaheinejad, R.C. Greenwood, N.R. Johnson, I.Y. Lee and F.K. McGowan, to be published.

6. A. Sandulescu, D.N. Poenaru and W. Greiner, Sov. J. Part. Nucl. 11(6):528 (1980).

7. A. C. Wahl, Atomic Data and Nuclear Data Tables 39:1 (1988).

8. J.B. Wilhelmy, et al., Phys. Rev. SC:204 (1972).

9. J.R. Leigh, et al., Phys. Lett. 159B:9 (1985).

10. Y. Abdelrahman, et al., Phys. Lett. 199B:504(1987).

11. M. Ogihara, et al., Z. Phys. A335:203 (1990).

12. J. Cizewski, M.A. C. Hotchkis, J. L. Durell, J. Copnell, A.S. Mowbray, J. Fitzgerald, W. R. Phillips, L. Ahmad, M.P. Carpenter, R.V.F. Janssens, T.L. Khoo, E.F. Moore, L.R. Morss, Ph. Benet, and D. Ye, Phys. Rev. C47:1294 (1993).

13. R. Aryaeinejad, J.D. Cole, R.C. Greenwood, S.S. Harrill, N.P. Lohsteter, K. ButlerMoore, J.H. Hamilton, A.V. Ramayya, X. Zhao, W.C. Ma, J.Kormicki, J.K. Deng, W. B. Gao, I.Y. Lee, N.R. Johnson, F.K. McGowan, G. Ter-Akopian, and Yu. Oganessian, Phys. Rev. C 48:566 (1993). 